\title{
An Update on Treatment Modalities for Ulnar Nerve Entrapment: A
}

\section{Literature Review}

\author{
Neeraj Vij (iD 1, ${ }^{*}$, Blake Traube ${ }^{1}$, Roy Bisht ${ }^{1}$, Ian Singleton ${ }^{1}$, Elyse M. Cornett ${ }^{2}$, Alan D. Kaye ${ }^{2}$, Farnad \\ Imani (iD ${ }^{3}$, Ali Mohammadian Erdi (iD ${ }^{4, * *}$, Giustino Varrassi (iD ${ }^{5}$, Omar Viswanath ${ }^{10}{ }^{1,6,7}$ and Ivan Urits \\ (iD) 8 \\ ${ }^{1}$ University of Arizona College of Medicine - Phoenix, Phoenix, Arizona \\ ${ }^{2}$ Louisiana State University Health Shreveport, Department of Anesthesiology, Shreveport, Louisiana \\ ${ }^{3}$ Pain Research Center, Department of Anesthesiology and Pain Medicine, Iran University of Medical Sciences, Tehran, Iran \\ ${ }^{4}$ Department of Anesthesiology, Ardabil University of Medical Sciences, Ardabil, Iran \\ ${ }^{5}$ Paolo ProcacciFoundation, Via Tacito 7, Roma, Italy \\ ${ }^{6}$ Creighton University School of Medicine, Department of Anesthesiology, Omaha, Nebraska \\ ${ }^{7}$ Valley Anesthesiology and Pain Consultants - Envision Physician Services, Phoenix, Arizona \\ ${ }^{8}$ Southcoast Health Physicians Group Pain Medicine, Wareham, Massachusetts \\ "Corresponding author: University of Arizona College of Medicine-Phoenix, 475 N 5th St, Phoenix, Arizona, 85004. Tel: 480-2986232. Email: neerajvij@email.arizona.edu \\ ***rresponding author: Department of Anesthesiology, Ardabil University of Medical Sciences, Ardabil, Iran. Email: a.mohammadian@arums.ac.ir
}

Received 2020 December 12; Accepted 2020 December 19.

\begin{abstract}
Context: Ulnar nerve entrapment is a relatively common entrapment syndrome second only in prevalence to carpal tunnel syndrome. The potential anatomic locations for entrapment include the brachial plexus, cubital tunnel, and Guyon's canal. Ulnar nerve entrapment is more so prevalent in pregnancy, diabetes, rheumatoid arthritis, and patients with occupations involving periods of prolonged elbow flexion and/or wrist dorsiflexion. Cyclists are particularly at risk of Guyon's canal neuropathy. Patients typically present with sensory deficits of the palmar aspect of the fourth and fifth digits, followed by motor symptoms, including decreased pinch strength and difficulty fastening shirt buttons or opening bottles.

Evidence Acquisition: Literature searches were performed using the below MeSH Terms using Mendeley version 1.19.4. Search fields were varied until further searches revealed no new articles. All articles were screened by title and abstract. Decision was made to include an article based on its relevance and the list of final articles was approved three of the authors. This included reading the entirety of the artice. Any question regarding the inclusion of an article was discussed by all authors until an agreement was reached. Results: X-ray and CT play a role in diagnosis when a bony injury is thought to be related to the pathogenesis (i.e., fracture of the hook of the hamate.) MRI plays a role where soft tissue is thought to be related to the pathogenesis (i.e., tumor or swelling.) Electromyography and nerve conduction also play a role in diagnosis. Medical management, in conjunction with physical therapy, shows limited promise. However, minimally invasive techniques, including peripheral percutaneous electrode placement and ultrasound-guided electrode placement, have all been recently studied and show great promise. When these techniques fail, clinicians should resort to decompression, which can be done endoscopically or through an open incision. Endoscopic ulnar decompression shows great promise as a surgical option with minimal incisions.

Conclusions: Clinical diagnosis of ulnar nerve entrapment can often be delayed and requires the suspicion as well as a thorough neurological exam. Early recognition and diagnois are important for early institution of treatment. A wide array of diagnostic imaging can be useful in ruling out bony, soft tissue, or vascular etiologies, respectively. However, clinicians should resort to electrodiagnostic testing when a definitive diagnois is needed. Many new minimally invasive techniques are in the literature and show great promise; however, further large scale trials are needed to validate these techniques. Surgical options remains as a gold standard when adequate symptom relief is not achieved through minimally invasive means.
\end{abstract}

Keywords: Ulnar Nerve Entrapment, Guyon's Canal Neuropathy, Ulnar Compression, Minimally Invasive, Surgical Ulnar Nerve, Non-Surgical Ulnar Nerve

\section{Context}

Localized nerve compression is common, and over the past 20 years, there has been an increase in the incidence of compressive neuropathy involving the upper $\operatorname{limb}(1,2)$. of the neuropathies involving the upper extremity, ulnar nerve entrapment is second only to median nerve entrap- 
ment at the carpal tunnel $(1,2)$. Though nervous injury in the upper limbs frequently affects patients who engage in recreational or occupational activities, it may also be caused by both acute elbow trauma and even ordinary activities such as habitually holding the arm in a flexed position while sleeping, leading to chronic compression neuropathy. Cubital tunnel syndrome, physical compression, or a scarring of the nerve in the setting of a fracture are all common causes $(3,4)$. Ulnar neuropathy is commonly encountered by clinicians in any medical setting; however, despite awareness of the condition and a plethora of research on the topic, diagnosis can be difficult (5). Nerve compression may mimic other common musculoskeletal disorders, and patients may not present until the symptoms are severe enough to cause significant sensory and motor functional deficits (6). This is significant as late presentation and diagnosis decreases the likelihood of full recovery and good clinical outcome $(5,6)$. Ulnar entrapment neuropathies can improve with conservative treatment including splinting, physical therapy, bracing, or injection, while others may require surgical intervention (1). Understanding of the nuances of clinical presentation and familiarity with the timing and types of surgical intervention is essential to the successful treatment of ulnar nerve entrapment (6). Therefore, it benefits clinicians to be familiar with ulnar nerve entrapment. This article offers a concise review of the pathophysiology, diagnosis, and treatment of ulnar nerve compression with a focus on conservative and minimally invasive interventions.

\section{Results}

\subsection{Risk Factors}

Risk factors for peripheral nerve compression include a superficial position of the nerve, course of a nerve that is long or in an area susceptible to trauma, paths through a notch or foramen (6). Intrinsic risk factors include smoking, educational level, and related working exeriences. Occupational factors include jobs that require task repetition, significant force or mechanical stresses, as well exposure to vibration and temperature (7).

In particular, ulnar nerve compression is common in patients who place the elbow or wrist in prolonged flexion and dorsiflexion respectively $(8,9)$. Flexion with the elbow while compressing against a surface especially magnifies the risk of cubital tunnel syndrome due to the increase in the intraneural pressure in this position (9). The existence of work-related musculoskeletal disorders such as medial epicondylitis, carpal tunnel syndrome, and radial tunnel syndrome have also be found to be risk factors (9). Cyclists and others who similarly have pressure place for a prolonged period on the hypothenar compartment of the hand are at an increased risk Guyon's canal neuropathy (compression of the ulnar nerve through guyon's canal) (2, 8).

Those with a significant varus or valgus deformity of the joint are also at an increased risk (9). Ulnar nerve irritation can also occur with medial collateral ligament deficiency (6). Recurrent subluxation of the nerve may also contribute to ulnar neuropathy due to the anterior roll over the medial epicondyle (3).

Trauma at the elbow can cause acute neuropraxia due to adjacent swelling of the subcutaneous tissues or displacement of fractures, and the subsequent scarring can result in a constriction of the nerve (3). In addition to the elbow, a similar pathology can occur with fractures near Guyon's canal (2).

The last source of injury to the ulnar nerve includes iatrogenic causes, which can occur during ligamentous reconstruction surgery such as Tommy John medial ulnar collateral ligament reconstruction (2). Anesthesized patients are also at risk for prolonged pressure on the ulnar nerve due to the postoperative position of the elbow in flexion (3).

\subsection{Clinical Presentation}

Patients with ulnar nerve compression typically present with paresthesias of the fourth and fifth digits $(1,2)$. Although sensory loss is typically the first symptom to be reported, the patient may also complain of an ill-defined upper extremity pain localized to the medial aspect of the elbow at the epicondylar groove in cases of ulnar neuropathy at the elbow $(5,9,10)$. Early symptoms typically occur intermittently and are often more severe at night, particularly if the flexion of the elbow occurs during sleeping (2). For patients with a high physical demand on their elbows, such as athletes or laborers, symptoms may be exacerbated by increased activity that places the ulnar nerve on stretch (2). As the disease progresses, the symptoms progress to occur more frequently and through all hours of the day. In these cases, prolonged elbow flexion may be able to provoke the patient's symptoms (2). However, patients with ulnar nerve entrapment often present with more advanced disease compared to patients with carpal tunnel syndrome (1). Patients with more prolonged compression can present with intrinsic muscle weakness causing weak grip, decreased pinch strength, fatigue, clumsiness of the hand, and difficulty with fine motor tasks such as opening bottles or buttoning $(1,5)$. In chronic cases, there may be marked wasting of the small muscles of the hand as well as in forearm muscles (9). Crossing the fingers may be difficult because of interosseous weakness, and patients may describe 
the fifth digit getting caught when placing the hand in a pocket (1). As in carpal tunnel syndrome, traditional provocative maneuvers for ulnar nerve entrapment are not sensitive or specific. Paresthesia caused by tapping over the ulnar nerve at the elbow can be induced in $34 \%$ of normal volunteers, and $20 \%$ endorse symptoms after 3 minutes of elbow flexion (1).

\subsection{Diagnosis}

Radiographs of the involved wrist or elbow should be obtained to determine if the ulnar nerve entrapment is due to a bony deformity or abnormality as Classic peripheral neuropathy may be caused by anatomic variations that are visible on plain radiographs $(5,6,9)$. For entrapment suspected at the wrist, carpal tunnel and oblique views should be obtained, and a computed tomography scan of the hand can be considered to rule out a fracture of the hook of the hamate $(5,7)$. Soft tissue anomalies, including a space-occupying lesion causing nerve compression, may be evident on magnetic resonance imaging such as thickening of the medial collateral ligament, ganglion cysts, accessory muscles, soft-tissue tumors, or aneurysms $(5,9,11-14)$. Ulnar nerve symptoms in the hand caused by thrombosis of the ulnar artery can be confirmed via the use of a duplex scan ultrasound or an angiogram $(5,8)$. Ultrasound of the cubital tunnel or elbow can be also be used to detect neuropathy, which presents initially as an abnormal enlargement of the nerve with decreased echogenicity, eventually causing the nerve to atrophy with a concurrent swelling proximal to the site of compression $(5,9,11,15)$.

Electrodiagnostic testing remains an important tool in diagnosing compression neuropathy with muscular denervation (5). Electromyography records the electrical activity of a muscle from which results in spontaneous action potential muscle fibrillations and decreased interference patterns with maximal voluntary effort $(5,6)$. Nerve conduction studies measure the amplitude, velocity, and latency of the muscular action potential (5). A patient with cubital tunnel syndrome may have the finding of decreased conduction velocity in a segment of the ulnar nerve that crosses the elbow while a conduction study can aid in localizing the specific area of compression at the elbow $(3,5)$. However, for most patients with a typical presentation of ulnar nerve entrapment, nerve conduction studies will not change the clinical course (6).

\subsection{Anatomy}

The ulnar nerve originates in the axilla as a continuation of the median cord in the brachial plexus, originally arising from $\mathrm{C} 8$ and $\mathrm{T} 1$ nerve roots in the spinal cord $(5,16$, 17). Distally, the ulnar nerve tracks down the arm in the medial neurovascular bundle, through the posterior compartment of the upper arm, posterior to the brachial artery. At the elbow joint, the ulnar nerve passes through the condylar grove of the cubital tunnel in the medial and posterior aspects of the medial condyle $(16,17)$. Within the forearm, the ulnar nerve travels posteriorly to the flexor carpi ulnaris muscle, which it also innervates. As it reaches the wrist, it produces the dorsal cutaneous branch, providing sensation to the dorsal ulnar aspect of the wrist $(16,17)$. The ulnar nerve enters Guyon's canal once it reaches the hand. Distal to Guyon's canal, the ulnar nerve branches into a superficial sensory branch and the deep motor branch $(5,16$, 17). The former provides sensation to the fourth and fifth fingers while the latter supplies the hypothenar muscles of the hand, the interosseous muscles, as well as the third and fourth lumbricals $(5,16,17)$.

Ulnar nerve entrapment is the second most common nerve entrapment in the body (18). Primarily, it becomes entrapped as it passes through the cubital tunnel at the elbow or Guyon's canal at the wrist $(17,18)$. In the cubital tunnel, this may be caused by either Osborne's ligament, which forms the roof of the tunnel, or the medial condyle. The arcade of Struthers is another potential ulnar nerve entrapment site; this is a filmy tissue located between the medial head of the triceps muscle and the medial intermuscular septum (4). In the distal forearm, ulnar nerve compression can be caused by deep fascia of the flexor carpi ulnaris or the fascia of the flexor digitorum superficialis (19).

\subsection{Pathophysiology}

Given the superficial position of the ulnar nerve within the ulnar groove, it is susceptible to external compression in certain positions, such as resting an elbow on an armchair (1). Elbow flexion significantly narrows the cubital tunnel, which results in ulnar nerve compression $(1,19)$. There has been a documented decrease of 30 - 41\% in the cross-sectional area within the cubital tunnel during elbow flexion (19). This has been accredited to the change of shape that occurs in the cubital tunnel during flexion, as it goes from an ovoid shape to a trapezoidal shape 20. During this process, the length of the ulnar nerve also increases in addition to the compression seen. As a result, physical activity involving repetitive flexion and extension movements of the elbow has been associated with an increased incidence of ulnar neuropathy $(1,19)$. Sonographic examinations have also shown that those with ulnar neuropathy demonstrate increased ulnar nerve displacement during elbow flexion and extension compared to controls (20).

At the wrist, one of the more common causes of ulnar nerve compression is the presence of a mass, particularly a ganglion cyst $(8,21)$. These cysts usually originate from 
the pisotriquetral, triquetrohamate, ulnocarpal, and midcarpal joints $(8,21)$. Other masses, including giant cell tumors, lipomas, intraneural cysts, nerve tumors, and vascular malformations, can also cause ulnar neuropathy but are less likely to do so $(8,21)$. Another major cause of ulnar nerve entrapment at the wrist is trauma from acute injuries or repetitive motions. Events such as jackhammering, cycling, weight lifting, or throwing have resulted in ulnar neuropathy $(8,21-23)$. Within trauma-related etiologies, the most commonly reported cause are disruptions of the hook of the hamate $(8,21)$. Other causes of ulnar nerve compression at the wrist include arthritic disease, Behcet disease, bony abnormalities, leprosy, and edema $(8,21)$.

\subsection{Medical Management}

The use of medical management is limited in ulnar nerve entrapment syndromes. Elhasan et al. in 2007 did report some success with non-surgical management, including NSAIDs in conjunction with a cushion or protective splinting 5. However, the wide majority of papers on the field do not allude to medical management alone as an option and speak to corticosteroid injections, a minimally invasive technique, as the next therapy when conservative techniques such as splinting and bracing fail $(1,8,16,24)$.

\subsection{Minimally Invasive Techniques}

Before any surgical intervention, patients can be treated with minimally invasive techniques in attempts to relieve an entrapped ulnar nerve. Peripheral percutaneous electrode placement has been studied and has shown potential in reducing pain symptoms. This technique, using a stimulating needle to place the electrode, proved to be a simple procedure that was effective in reducing pain symptoms, with the best results seen at a slow frequency and intermittent stimulation (25). All of the five patients followed in a case series had an excellent pain response and improved quality of life, and after the six-year follow-up, the first patient still had the electrode in place (25). Peripheral nerve stimulation can also be applied without the use of permanent electrodes. The use of an anesthetic solution and applicator of a nerve stimulator to the sensory or combined sensory and motor nerves of the median and ulnar nerve resulted in successful nerve blocks (26). Pressure monitoring during peripheral nerve injections can be an effective method to assess safety and efficacy during nerve blockade (27). Pressure measurements on average for ulnar nerve injections were $17.9 \mathrm{psi}$ and $6.7 \mathrm{psi}$ for intraneural and perineural injections, respectively (27). Injection pressures over 25 psi have resulted in persistent motor defects, so monitoring these pressure changes during injections can greatly reduce the incidence of permanent nerve damage.
Ultrasound-guided techniques have also been studied to assess their utility in correcting ulnar nerve entrapment. One study examined the ability of ultrasound in placing targeted electrodes on radial, median, and ulnar nerves in the distal upper extremity on cadavers (28). Electrodes that had been targeted to the ulnar nerve had been placed 9 to $13 \mathrm{~cm}$ above the medial epicondyle showed no migration after 21 hours of continuous passive motion, which was used to stimulate activity (28). This method was also studied in a cohort of eight living patients who underwent ultrasound-guided through-the-needle placement of percutaneous neurostimulation electrodes in the ulnar, radial, median, peritoneal, and posterior tibial nerves (29). The overall results in the study were promising, with seven out of nine peripheral nerves having a successful trial with permanent electrode implantation. The only ulnar nerve patient that followed up throughout the study showed an $80 \%$ reduction in medication usage before infection resulted in explantation (29). While ultrasound has a potential benefit in treating neuropathy from ulnar nerve entrapment, there are some limitations. In addition to showing limited capability in determining the potential for success and grade of peripheral nerve tumors, it is difficult to distinguish between nerve injuries that are self-hearing and those that will eventually require surgical intervention (12).

Ultimately, the option of performing a minimally invasive procedure depends on the severity of the ulnar nerve entrapment. Evidence has shown that the degree of compression correlates to whether minimally invasive techniques will be successful. A review covering 50 papers with over 2000 patients suffering from ulnar nerve entrapment found that approximately half of the patients with minimal ulnar nerve compression experienced recovery without any operative procedures (30). Conversely, this review found that the use of nonoperative measures was completely unsuccessful in treating severe ulnar nerve compression (30).

\subsection{Surgical Techniques}

When minimally invasive techniques are ineffective, or symptoms become progressively more severe, surgical treatment is indicated, as the benefit outweighs the risk of vascular and nerve damage (8). Surgical intervention should be patient-tailored, as there are multiple sites of possible ulnar nerve compression along its course. The general goal of these techniques is total decompression of the nerve and placement into a region without risk of compression or kinking. Two primary approaches are frequently discussed - simple decompression, where adhesions and fibers constricting the nerve are cut without relocation of the ulnar nerve, and decompression with trans- 
position of the ulnar nerve. Medial epicondylectomy is also an option, though pursued less often $(18,31)$. Additionally, many less invasive techniques for nerve decompression with and without transposition are under review, such as endoscope-assisted and in-situ small-incision procedures $(32,33)$.

A 2005 study compared traditional subcutaneous anterior transposition, where the ulnar nerve is moved from posterior medial epicondyle to anterior elbow, and traditional simple decompression. Of 66 total participants, 34 underwent anterior transposition, and 32 underwent simple decompression. At 3 and 9 months postoperatively, there was no significant difference in pain, sensorimotor deficits, and nerve conduction velocities between the two groups. Ultimately simple decompression was recommended as it is less invasive (34). However, if ulnar nerve subluxation is observed during the decompression procedure, a transposition is always indicated (32).

Endoscope-assisted ulnar nerve decompression is another less invasive intervention to be considered, whereby the division of fascia is carried out with a scope-knife under endoscope guidance. Endoscope-assisted transposition and/or medial epicondylectomy can also be added if ulnar nerve subluxation is revealed $(32,35,36)$.

A 2005 study tested the use of endoscopically assisted ulnar nerve decompression with a guiding-dissecting tool 38. In all, 36 patients underwent the procedure, with a mean follow-up of 14 months. In 21 patients, there no significant resideual motor or sensory symptoms. In 12 patients, there was an improvement from baseline, but with some persistence of sensory or motor syptoms or pain at the surgical site (37).

A 2007 - 2009 study of 46 patients undergoing endoscope-assisted cubital tunnel release, with a mean postoperative follow-up time of 5.1 months, resulted in no nerve injuries or recurrence of symptoms across all participants. One anterior transposition and one medial epicondylectomy were also performed at the time of decompression, without any significant sequelae (32). Another Orthopedic group at the University of Adelaide conducted 11 such surgeries from 2011-2014, with no major complications or recurrence of symptoms (33). This suggests much promise to less invasive endoscope-guided surgical techniques. However, long-term studies of the efficacy are still in short supply, and this procedure is contraindicated, in preference for the traditional open procedure, in patients with elbow deformity, elbow osteoarthritis, recurrent cubital tunnel, and other elbow pathology, decreasing the utility overall $(32,33)$.

Another less invasive surgical technique understudy is decompression with a small incision. This variation of traditional decompression uses a $1.5-2.0 \mathrm{~cm}$ incision be- tween the medial epicondyle and olecranon, compared to traditional $7.0 \mathrm{~cm}$ incisions or $0.5 \mathrm{~cm}$ endoscopic incisions, primarily to avoid scar pain, scar size, and numbness risks with the traditional method, and the special required training and difficulty with transposition with endoscopic procedures. One study looked at 51 patients undergoing ulnar nerve decompression with anterior transposition via a small incision, and all had improvements in grip and pinch strength and 2-point discrimination, without any scar pain or sensitivity, or residual numbness (33). Another study looked at in situ decompression at the site of the incision via a small incision method and found similar success, as this in-situ technique resulted in the resolution or improvement of symptoms in all patients without any complications (38).

There are multiple surgical approaches to the same mechanical need for decompression and occasionally transposition of the ulnar nerve in cases of ulnar nerve entrapment refractory to less invasive techniques. Studies show similar efficacy between the traditional, endoscopic, and small-incision procedures, and the decision of technique should be made on a case by case basis, with location (s) of compression, contraindications, and the presence or absence of subluxation as the primary determining factors.

\section{Conclusions}

Entrapment of the ulnar nerve is the second most common compressive neuropathy behind carpal tunnel syndrome. It can be caused by trauma, repetitive activities, or ordinary activities of daily living. Other risk factors include pregnancy, diabetes, and rheumatoid arthritis. Diagnosis is often late, decreasing the likelihood of full recovery. Early recognition and diagnosis are important for early institution of treatment. Typical symptoms for health care providers to recognize include paraesthesias of the small finger and ulnar aspect of the ring finger, poorly-defined medial elbow pain, and in more advanced disease, weakness of intrinsic hand muscles. Symptoms are often worse at night and may be provoked by elbow flexion. Radiographs, MRI, and ultrasound can rule out bony, soft tissue, and vascular etiologies, respectively. Definitive diagnosis can be accomplished with electrodiagnostic testing to find characteristic changes in nerve conduction velocity and amplitude, though testing will not often change management in patients with the characteristic presentation.

Ulnar nerve entrapment typically occurs at the cubital tunnel, though it may occur in the upper arm and distal forearm. The superficial position of the nerve predisposes it to compressive forces. Treatment is important to prevent further deterioration of the sensorimotor function of the ulnar nerve and proceeds from conservative 
to invasive modalities. Peripheral percutaneous electrode placement is one conservative measure effective in reducing pain symptoms via nerve stimulation, and electrodes also aid safer and more accurate anesthetic nerve blocks. Ultrasound-guided techniques allow more precise placement of electrodes as well, with strong efficacy. Although nearly half of patients with mild ulnar nerve entrapment find these conservative treatments sufficient, surgical intervention is needed in others. Surgical intervention primarily aims to decompress the nerve and, if necessary, transpose the nerve to a less compressive location. Traditional methods with larger incisions are effective, but more recent studies have shown similar efficacy can be found in less invasive surgical techniques for decompression and/or transposition, such as those conducted via small-incision or with the aid of an endoscope. Ulnar nerve entrapment is a common problem with many effective treatments, including both conservative and invasive treatments. Symptoms can be limited if detected early enough.

\section{Footnotes}

Authors' Contribution: Study concept and design: N. V. and I. U.; Acquisition of data: N. V. and I. U.; Analysis and Interpretation of data: N. V., B. T., R. B., I. S., E. C., A. K., F. I., G. V., A.M., O. V., I.U.; Drafting of the Manuscript: N.V., B. T., R. B., I.S., E. C., A. K., F. I., G. V., A.M., O. V., I. U.; critical revision of the manuscript for important intellectual content: N.V., B. T., R. B., I. S., E. C., A. K., F. I., G. V., A.M., O. V., I. U.; Statistical Analysis: none; Administrative, technical, and material support: N. V. and I. U.; Study supervision: N.V. and I. U.

Conflict of Interests: We have no conflicts of interest. Funding/Support: We did not receive any funding/support for this project.

\section{References}

1. Doughty CT, Bowley MP. Entrapment neuropathies of the upper extremity. Med Clin North Am. 2019;103(2):357-70. doi: 10.1016/j.mcna.2018.10.012. [PubMed: 30704687].

2. Dy CJ, Mackinnon SE. Ulnar neuropathy: evaluation and management. Curr Rev Musculoskelet Med. 2016;9(2):178-84. doi:10.1007/s12178016-9327-x. [PubMed: 27080868]. [PubMed Central: PMC4896870].

3. Dawson DM. Entrapment neuropathies of the upper extremities. $N$ Engl J Med. 1993;329(27):2013-8. doi: 10.1056/NEJM199312303292707. [PubMed: 8247077].

4. Campbell WW, Landau ME. Controversial entrapment neuropathies. Neurosurg Clin N Am. 2008;19(4):597-608. vi-vii. doi: 10.1016/j.nec.2008.07.001. [PubMed: 19010284].

5. Elhassan B, Steinmann SP. Entrapment neuropathy of the ulnar nerve. J Am Acad Orthop Surg. 2007;15(11):672-81. doi: 10.5435/00124635200711000-00006. [PubMed: 17989418].

6. Neal S, Fields KB. Peripheral nerve entrapment and injury in the upper extremity. Am Fam Physician. 2010;81(2):147-55. [PubMed: 20082510].
7. Szabo RM, Steinberg DR. Nerve entrapment syndromes in the wrist. J Am Acad Orthop Surg. 1994;2(2):115-23. doi: 10.5435/00124635199403000-00005. [PubMed: 10708999].

8. Earp BE, Floyd WE, Louie D, Koris M, Protomastro P. Ulnar nerve entrapment at the wrist. J Am Acad Orthop Surg. 2014;22(11):699-706. doi: 10.5435/JAAOS-22-11-699. [PubMed: 25344595].

9. Cutts S. Cubital tunnel syndrome. Postgrad Med J. 2007;83(975):2831. doi: 10.1136/pgmj.2006.047456. [PubMed: 17267675]. [PubMed Central: PMC2599973].

10. Hobson-Webb LD, Juel VC. Common entrapment neuropathies. Continuum (Minneap Minn). 2017;23(2, Selected Topics in Outpatient Neurology):487-511. doi: 10.1212/CON.0000000000000452. [PubMed: 28375915].

11. Martinoli C, Bianchi S, Pugliese F, Bacigalupo L, Gauglio C, Valle $\mathrm{M}$, et al. Sonography of entrapment neuropathies in the upper limb (wrist excluded). J Clin Ultrasound. 2004;32(9):438-50. doi: 10.1002/jcu.20067. [PubMed: 15558622].

12. Ray WZ, Mahan MA, Guo D, Guo D, Kliot M. An update on addressing important peripheral nerve problems: challenges and potential solutions. Acta Neurochir (Wien). 2017;159(9):1765-73. doi: 10.1007/s00701017-3203-3. [PubMed: 28500566].

13. Kim S, Choi JY, Huh YM, Song HT, Lee SA, Kim SM, et al. Role of magnetic resonance imaging in entrapment and compressive neuropathywhat, where, and how to see the peripheral nerves on the musculoskeletal magnetic resonance image: part 2. Upper extremity. Eur Radiol. 2007;17(2):509-22. doi: 10.1007/s00330-006-0180-y. [PubMed: 16572333].

14. Andreisek G, Crook DW, Burg D, Marincek B, Weishaupt D. Peripheral neuropathies of the median, radial, and ulnar nerves: MR imaging features. Radiographics. 2006;26(5):1267-87. doi: 10.1148/rg.265055712. [PubMed: 16973765].

15. Lin C, Chen Y, Chen W. Application of ultrasound and ultrasoundguided intervention for evaluating elbow joint pathologies. J Med Ultrasound. 2012;20(2):87-95. doi: 10.1016/j.jmu.2012.04.007.

16. Pratt N. Anatomy of nerve entrapment sites in the upper quarter. $J$ Hand Ther. 2005;18(2):216-29. doi: 10.1197/j.jht.2005.02.004. [PubMed: 15891980].

17. Jacobson JA, Fessell DP, Lobo Lda G, Yang LJ. Entrapment neuropathies I: upper limb (carpal tunnel excluded). Semin Musculoskelet Radiol. 2010;14(5):473-86. doi: 10.1055/s-0030-1268068. [PubMed: 21072726].

18. Arle JE, Zager EL. Surgical treatment of common entrapment neuropathies in the upper limbs. Muscle \& Nerve. 2000;23(8):1160-74. doi: 10.1002/1097-4598(200008)23:8<1160::aid-mus2>3.0.co;2-4.

19. Staples JR, Calfee R. Cubital tunnel syndrome: Current concepts. JAm Acad Orthop Surg. 2017;25(10):e215-24. doi: 10.5435/JAAOS-D-15-00261. [PubMed: 28953087].

20. Yang SN, Yoon JS, Kim SJ, Kang HJ, Kim SH. Movement of the ulnar nerve at the elbow: a sonographic study. J Ultrasound Med. 2013;32(10):1747-52. doi: 10.7863/ultra.32.10.1747. [PubMed: 24065255].

21. Coraci D, Loreti C, Piccinini G, Doneddu PE, Biscotti S, Padua L. Ulnar neuropathy at wrist: entrapment at a very "congested" site. Neurol Sci. 2018;39(8):1325-31. doi: 10.1007/s10072-018-3446-7. [PubMed: 29779137].

22. Aoki M, Takasaki H, Muraki T, Uchiyama E, Murakami G, Yamashita T. Strain on the ulnar nerve at the elbow and wrist during throwing motion. J Bone Joint Surg Am. 2005;87(11):2508-14. doi: 10.2106/JBJS.D.02989. [PubMed: 16264128].

23. Depukat P, Mizia E, Kuniewicz M, Bonczar T, Mazur M, Pelka P, et al. Syndrome of canal of Guyon - definition, diagnosis, treatment and complication. Folia Med Cracov. 2015;55(1):17-23. [PubMed: 26774628].

24. Filippi R, Farag S, Reisch R, Grunert P, Bocher-Schwarz H. Cubital tunnel syndrome. Treatment by decompression without transposition of ulnar nerve. Minim Invasive Neurosurg. 2002;45(3):164-8. doi: 10.1055/s2002-34394. [PubMed: 12353165]. 
25. Kothari S, Goroszeniuk T. Percutaneous permanent electrode implantation to ulnar nerves for upper extremity chronic pain: 6 years follow up. Reg Anesth Pain Med. 2006;31(5):16. doi: 10.1016/j.rapm.2006.06.211.

26. Macaire P, Choquet O, Jochum D, Travers V, Capdevila X. Nerve blocks at the wrist for carpal tunnel release revisited: The use of sensorynerve and motor-nerve stimulation techniques. Reg Anesth Pain Med. 2005;30(6):536-40. doi: 10.1016/j.rapm.2005.06.010. [PubMed: 16326338].

27. Krol A, Szarko M, Vala A, De Andres J. Pressure monitoring of intraneural an perineural injections into the median, radial, and ulnar nerves; lessons from a cadaveric study. Anesth Pain Med. 2015;5(3). e22723. doi: 10.5812/aapm.22723. [PubMed: 26161318]. [PubMed Central: PMC4493739].

28. Huntoon MA, Hoelzer BC, Burgher AH, Hurdle MF, Huntoon EA. Feasibility of ultrasound-guided percutaneous placement of peripheral nerve stimulation electrodes and anchoring during simulated movement: part two, upper extremity. Reg Anesth Pain Med. 2008;33(6):55865. doi: 10.1016/j.rapm.2008.04.006. [PubMed:19258971].

29. Huntoon MA, Burgher AH. Ultrasound-guided permanent implantation of peripheral nerve stimulation (PNS) system for neuropathic pain of the extremities: original cases and outcomes. Pain Med 2009;10(8):1369-77. doi: 10.1111/j.1526-4637.2009.00745.x. [PubMed 20021597].

30. Lee Dellon A. Review of treatment results for ulnar nerve entrapment at the elbow. J Hand Surg. 1989;14(4):688-700. doi: 10.1016/03635023(89)90192-5.

31. Huang JH, Samadani U, Zager EL. Ulnar nerve entrapment neuropathy at the elbow: simple decompression. Neurosurgery. 2004;55(5):1150-3. doi: 10.1227/01.neu.0000140841.28007.f2. [PubMed: 15509321].

32. Mirza A, Reinhart MK, Bove J, Litwa J. Scope-assisted release of the cubital tunnel. J Hand Surg Am. 2011;36(1):147-51. doi: 10.1016/j.jhsa.2010.10.016. [PubMed: 21193135].

33. Zhang X, Dhawan V, Yu Y, Zhang G, Shao X. A minimally invasive approach for cubital tunnel release and ulnar nerve transposition. Phys Sportsmed. 2017;45(2):110-3. doi: 10.1080/00913847.2017.1295775. [PubMed: 28276992].

34. Nabhan A, Ahlhelm F, Kelm J, Reith W, Schwerdtfeger K, Steudel WI. Simple decompression or subcutaneous anterior transposition of the ulnar nerve for cubital tunnel syndrome. J Hand Surg Br. 2005;30(5):521-4. doi:10.1016/j.jhsb.2005.05.011. [PubMed: 16061314].

35. Morse LP, McGuire DT, Bain GI. Endoscopic ulnar nerve release and transposition. Tech Hand Up Extrem Surg. 2014;18(1):10-4. doi: 10.1097/BTH.0000000000000030. [PubMed: 24296546].

36. Orhurhu V, Orman S, Peck J, Urits I, Orhurhu MS, Jones MR, et al. Carpal tunnel release surgery- A systematic review of open and endoscopic approaches. Anesth Pain Med. 2020;10(6). 112291. doi: 10.5812/aapm.112291.

37. Ahcan U, Zorman P. Endoscopic decompression of the ulnar nerve at the elbow. J Hand Surg Am. 2007;32(8):1171-6. doi: 10.1016/j.jhsa.2007.07.004. [PubMed: 17923299].

38. Koehler SM, Lovy AJ, Guerra SM, Chawla H, Hausman MR. Minimally invasive in situ cubital tunnel decompression. Tech Hand Up Extrem Surg. 2016;20(1):42-7. doi: 10.1097/BTH.0000000000000113. [PubMed: 26829676]. 